\title{
HUBBLE SPACE TELESCOPE PHOTOMETRY OF THE CENTRAL REGIONS OF VIRGO CLUSTER ELLIPTICAL GALAXIES. I. OBSERVATIONS, DISCUSSION, AND CONCLUSIONS
}

\author{
WALTER JAFFE \\ Leiden Observatory, Leiden, The Netherlands \\ Electronic mail: jaffe@strw.leidenuniv.nl \\ HOLLAND C. FORD \\ Johns Hopkins University, Space Telescope Science Institute, Baltimore, Maryland 21218 \\ Electronic mail: ford@jhufos.pha.jhu.edu \\ ROBERT W. O'CONNELL \\ University of Virginia, Charlottesville, Virginia 22903 \\ Electronic mail: rwo@perseus.astro.virginia.edu \\ FRANK C. VAN DEN BOSCH \\ Leiden Observatory, Leiden, The Netherlands \\ Electronic mail: vdbosch@strw.leidenuniv.nl \\ LAURA FERRARESE \\ Johns Hopkins University, Space Telescope Science Institute, Baltimore, Maryland 21218 \\ Electronic mail: ferrares@jhufos.pha.jhu.edu \\ Received 1994 March 9; revised 1994 June 17
}

\begin{abstract}
Using the Hubble Space Telescope we have observed at $10 \mathrm{pc}$ resolution the nuclei of a luminosity-limited sample of $14 \mathrm{E}$ and $\mathrm{E} / \mathrm{S} 0$ galaxies in the Virgo Cluster with magnitudes $B_{T}=9.4$ to 13.4 . In this paper we present the images, and discuss the results of the detailed analysis of the surface photometry given in two companion papers. We find that the nuclear and near-nuclear morphologies confirm and strengthen the previously recognized dichotomy of "E" galaxies into "true" and "disky" subtypes. The latter, usually classified E4 or later, often show a bright nuclear disk of radius $\sim 100 \mathrm{pc}$. Essentially all early-type galaxies with $-18>M_{B}>-20$ are disky. Most true E galaxies are classified E4 or earlier. Most galaxies of both types show dust in the nuclear regions, the most remarkable example being a compact dust disk in NGC 4261. Other than dust, no anomalies were detected in the centers of the three galaxies in our sample which show clear kinematic evidence for a decoupled component.
\end{abstract}

\section{INTRODUCTION}

Numerous studies over the last decade have revealed elliptical galaxies to be far more complex than their outwardly bland and symmetrical morphologies would suggest. Rather than the ancient, homogeneous, and almost purely stellar systems which were anticipated from the traditional picture developed by Hubble and Baade, they are now often found to contain a substantial interstellar medium, multiple, discrete dynamical components, and stellar populations with a wide range of ages. Much, though not all, of this complexity apparently originates in tidal encounters, accretion events, mergers, or other interactions between galaxies and their environments. Recent reviews on the structure of ellipticals, their interstellar media, their stellar populations, and the role which may be played by interactions can be found in Kormendy \& Djorgovski (1989), Roberts et al. (1991), O'Connell (1994), and Barnes \& Hernquist (1992), respectively. Additionally, the statistical frequency of active nuclei in the form of QSOs and radio galaxies at higher redshifts led to the realization that most nearby ellipticals should har- bor massive black holes, if these are indeed the engines which drive nuclear activity (e.g., Rees 1984; Kormendy 1993). These central masses may contribute to the morphological complexity of their hosts.

The properties of the outer envelope of an elliptical galaxy are not as strongly affected by its evolutionary history as its nuclear regions. At the distance of the Virgo Cluster, for instance, it would not be possible to distinguish the signature of a black hole from that of a recent merger at radii larger than about $2^{\prime \prime}$, whereas the photometric and kinematic characteristics at smaller radii would be very different in the two cases (Young 1980; Kormendy 1984). The centers of ellipticals are therefore of primary interest.

The Hubble Space Telescope (HST), even without optical correction for the spherical aberration of its primary mirror, offered important capabilities to study the structure of the centers of elliptical galaxy on scales not accessible with ground-based telescopes. Because of the strong central peak of the point spread function (PSF) and the stable optical characteristics of the Telescope, image reconstruction algorithms are able to provide considerable information on scales 
of $\$ 0$ ".2 (White \& Allen 1990). HST has been used by several groups to observe at high resolution a number of elliptical galaxies with known intriguing optical, radio, $\mathrm{x}$-ray, or infrared characteristics. However, it seemed desirable to us to perform an $H S T$ survey on a sample of galaxies whose selection was unbiased with regard to such abnormalities. From this kind of sample we can begin to investigate the frequency with which encounters or black holes, for instance, influence the evolution of galaxies.

Accordingly, we have begun a survey of elliptical galaxies, chosen solely on the basis of their luminosity in the $B$ band, in the Virgo Cluster, which is the nearest grouping containing a large sample. The first phase of our survey, which is presented in this paper, has concentrated on obtaining F555W ( $V$ band) Planetary Camera (PC) images of the galaxy nuclei, with which to investigate their central structure. We are primarily interested in determining surface brightness profiles and isophotal shapes at high resolution, with emphasis on the statistics of abnormalities such as unresolved cores, strong . isophotal twists, dust lanes, and nuclear disks. The presence of unresolved nuclear structures on HST PC and Faint Object Camera images has already greatly strengthened the case for massive black holes in the Local Group Sb galaxy M31 (Lauer et al. 1993) and the dwarf elliptical M32 (Lauer et al. 1992a), and in the Fornax cluster elliptical NGC 1399 (Stiavelli et al. 1993). However, an unambiguous demonstration of the presence of a black hole demands kinematical information on the same spatial scales (Kormendy 1993), and this requires the use of the $H S T /$ COSTAR correcting optics.

In this paper we describe the observations, data reduction, and analysis procedures and present the overall statistical results of our survey. Subsequent papers (van den Bosch et al. 1994; Ferrarese et al. 1994, hereafter referred to as Papers II and III, respectively) will discuss in more detail the isophotal shapes and the brightness profiles, respectively. The outline of this paper is as follows: in Sec. 2 we describe the selection of our sample; Section 3 describes the data reduction procedures, image reconstruction methods, and analysis technique; Section 4 presents the images, and discusses those features immediately visible. Section 5 summarizes the results of Papers II and III and discusses their broader implications. Section 6 contains our conclusions.

A note on terminology: in this paper galactic nucleus refers to regions unresolved by the $H S T$ at Virgo, i.e. $<10$ pc; nuclear regions or central regions refer to a radius of approximately $300 \mathrm{pc}$ around the nucleus, and core refers to a region surrounding the nucleus where the brightness profile is much flatter than at larger radii.

\section{THE SAMPLE}

We selected a statistically complete $B$ magnitude limited sample of Virgo objects classified as E or E/S0 in the RSA (Revised Shapley-Ames Catalog: Sandage \& Tammann 1981). Cluster membership criteria are described by Huchra (1984). The relative proximity of the Virgo cluster (14.7 Mpc; Jacoby et al. 1990) permits relatively high spatial resolution. In addition, since all the galaxies are at approximately the same distance, results can be compared without the complications of differing spatial resolution or uncertain distance estimates. NGC 4486 (M87) and NGC 4472 (M49) were not included in our original program since they had already been observed by other investigators. PC images for these are now in the $H S T$ public archive, and we have therefore added them to the sample. The data for NGC 4486 was derived from HST program 3197 (H. C. Ford, Principal Investigator), and for NGC 4472 from program 3229 (J. Westphal, PI). The image of NGC 4486 is of lower signal/noise ratio than an image previously published (Lauer et al. 1992b) but was used because the PSF of that image is poorly defined. Three of the luminous galaxies in Virgo (NGC 4406, NGC 4621, and NGC 4660), which would otherwise have been included, could not be observed with HST because of a lack of suitable guide stars. Their elimination is thus unbiased with respect to morphology, magnitude, or any other intrinsic property. With the exception of these three, the sample includes all Virgo elliptical galaxies brighter than $B_{T}=13.4$.

The final sample is listed in Table 1 in order of decreasing $B$ band luminosity. The table includes morphological classifications, integrated magnitudes, far infrared, $x$-ray, and radio fluxes.

\section{OBSERVATIONS AND DATA ANALYSIS PROCEDURES}

\subsection{Observations and Data Reduction}

HST images of the 12 galaxies in our observing program, HST 2607, were obtained between 1991 February 24 and 1993 March 15 with the PC using the wide band filter F555W, which corresponds closely to the Johnson/Cousins $V$ band. The PC consists of a mosaic of four $800 \times 800 \mathrm{CCD}$ detectors. Each pixel $(15 \mu \mathrm{m})$ subtends 0."043, corresponding to $3.1 \mathrm{pc}$ at the distance of the Virgo cluster. The total field of view of the camera is $1.1 \times 1.1$, or $4.9 \times 4.9 \mathrm{kpc}$. A detailed description of the PC can be found in the HST/WFPC Instrument Handbook, MacKenty et al. (1992). The galaxy centers were placed on PC chip 6. All observations were obtained with the telescope guiding in coarse track, which typically gives an rms tracking error of 0.015 .

All of the images were recalibrated during July 1993 using standard methods recommended by the WF/PC Instrument Definition Team (see Lauer 1989) and the most up-todate reference files. Briefly, five calibration steps were performed in the following order: statistical correction for errors in the analog-to-digital (A to D) conversion, bias subtraction, preflash subtraction, dark subtraction, and flatfield division. The A to D correction removes the systematic degradation introduced by the conversion electronics. The bias correction removes the background produced by the electronic bias by subtracting the average of a set of individual bias readouts. To correct for the uneven illumination pattern of the preflash image, a large number of preflash exposures are averaged, scaled by the preflash exposure time, and subtracted from the science image. The dark frame is constructed from orbit-long dark exposures; the nominal value for the CCD dark current is $0.003 \mathrm{e} / \mathrm{pixel} / \mathrm{s}$. Finally division by the mean flatfield frame corrects for variations in sensi- 
TABLE 1. The sample.

\begin{tabular}{|c|c|c|c|c|c|c|}
\hline $\begin{array}{c}\text { Galaxy } \\
\text { (1) }\end{array}$ & $\begin{array}{c}\text { RSA } \\
(2)\end{array}$ & $\begin{array}{c}\mathrm{RC} 2 \\
(3)\end{array}$ & $\begin{array}{l}B_{T} \\
(4)\end{array}$ & $S_{100 \mu \mathrm{m}}$ & $\begin{array}{l}S_{21 \mathrm{~cm}} \\
(6)\end{array}$ & $\begin{array}{l}F_{\mathbf{X}} \\
(7)\end{array}$ \\
\hline NGC 4472 & $\mathrm{E} 1 / \mathrm{S}_{1}$ & E2 & 9.37 & $0 \pm 90$ & 225 & $837 \pm 20$ \\
\hline NGC 4486 & E0 & E0pec & 9.59 & $360 \pm 90$ & 214000 & $50000^{*}$ \\
\hline NGC 4374 & $\mathrm{E} 1$ & E1 & 10.09 & $1030 \pm 110$ & 6100 & $154 \pm 16$ \\
\hline NGC 4365 & E3 & E3 & 10.52 & $580 \pm 120$ & $<4$ & $24 \pm 6$ \\
\hline NGC 4473 & E5 & E5 & 11.16 & $0 \pm 95$ & $<6$ & $13 \pm 9$ \\
\hline NGC 4261 & E3 & E3 & 11.41 & $130 \pm 40$ & 18600 & $92 \pm 13$ \\
\hline NGC 4570 & $\mathrm{~S}_{1} / \mathrm{E} 7$ & So & 11.84 & $0 \pm 100$ & $<10$ & \\
\hline NGC 4564 & E6 & E6 & 12.05 & $0 \pm 170$ & $<10$ & $5 \pm 6$ \\
\hline NGC 4168 & E2 & E2 & 12.11 & $590 \pm 140$ & $<11$ & $29 \pm 6$ \\
\hline NGC 4478 & E2 & E2 & 12.36 & $0 \pm 65$ & $<450$ & $-23 \pm 17$ \\
\hline NGC 4550 & $\mathrm{E} 7 / \mathrm{S}_{1}$ & SB0 & 12.56 & $220 \pm 80$ & $<10$ & $3 \pm 5$ \\
\hline NGC 4476 & E5pec & $\mathrm{SO}^{-}$ & 13.01 & $1640 \pm 95$ & $<450$ & $-2 \pm 12$ \\
\hline NGC 4623 & E7 & SB0+ & 13.24 & $0 \pm 105$ & $<10$ & \\
\hline NGC 4342 & E7 & $\mathrm{S}^{-}$ & 13.41 & $0 \pm 160$ & $<3$ & \\
\hline
\end{tabular}

Notes to TABLE 1

Notes: Column (1) gives the NGC name of the galaxy. Columns (2) and (3) give the ellipticity class and type according to the Revised Shapley-Ames Catalog (RSA; Sandage \& Tammann 1981) and the Second Reference Catalog (RC2; de Vaucouleurs et al. 1976), respectively. Column (4) presents the apparent total blue magnitude according to the RSA. Column (5) gives the IRAS flux density and rms noise in mJy at $100 \mu \mathrm{m}$ (Knapp et al. 1989). Column (6) gives the flux density at $20 \mathrm{~cm}$ in mJy (Wrobel, 1991). Column (7) gives the x-ray flux and error in the energy range $0.5-4.5 \mathrm{keV}$ in units of $10^{-14} \mathrm{erg} \mathrm{cm}^{-2} \mathrm{~s}^{-1}$ (Roberts et al. 1991).

*The $x$-ray flux from M87 is confused with that of the cluster cooling flow. The value quoted is from Fabricant et al. (1980) in the $0.2-3.0 \mathrm{keV}$ range.

tivity between pixels in the CCDs. Flatfields were obtained by observing the bright Earth surface as it passed below the spacecraft.

For our program, particular attention must be paid to the spurious effects of contaminants (MacKenty et al. 1992), which can affect the images in two ways: first by varying the level of the flatfield exposure with time, and second by creating spurious features (known as "measles") across the chips. In addition, decontamination procedures, which are executed to reduce the effects of contaminants, affect the large scale structure of the PC flatfields. The external Earth flatfield used to calibrate the images was obtained during December 1991, before any of the decontamination procedures were executed (February 1992, March 1992, and August 1992). Nevertheless, variations in the flatfield large scale structure as a consequence of decontamination can be corrected for by using internal lamp flatfields which, unlike the external flatfields, have been taken both before and after decontamination procedures. All science observations executed after a decontamination procedure were multiplied by an internal flatfield taken at the same epoch and divided by an internal flatfield taken prior to decontamination, i.e., at the same time as the external flatfield used for calibration. This procedure corrects for variations in the large scale structure of the flatfield but unfortunately does not remove the measles. The measles consist of roughly circular features at a few percent of the signal level, normally about 20 pixels $(0.9)$ in diameter, with darker centers and brighter outer rings. Since measles have been shown to be stable in the period when the images discussed here were taken, the ratio of internal flatfields, before and after decontamination, can be used as "measles maps." The measles could then be identified and removed by manually editing the images.

We have reduced only the PC CCD 6 frames on which the center of the galaxy was located. Two back-to-back exposures of 700 and $1700 \mathrm{~s}$, respectively, were taken for each galaxy, in order to facilitate the removal of cosmic rays. For NGC 4472 , three separate exposures were available in the archive $(160,800$, and $800 \mathrm{~s})$, while only a single exposure of NGC 4486 was available. For each galaxy, except NGC 4342, NGC 4486, NGC 4564, and NGC 4570, the multiple exposures were combined, using standard IRAF routines, and all pixels deviating more than $3 \sigma$ from the median were rejected. The $\sigma$ is evaluated from a noise model by finding the optimal quadratic sum of the photon noise, the readout noise, and a "scale" noise that depends linearly upon the median value. The resulting image was then edited in order to remove coincident cosmic ray events or cosmic rays that were overlooked by the IRAF routine. A different approach was necessary for NGC 4342, NGC 4564, and NGC 4570, for which the longer exposure was saturated in the inner 0.6. For these three objects, only the $700 \mathrm{~s}$ exposure has been used, and the cosmic rays have been removed by standard automated IRAF routines or by manually editing the images. The same was done for NGC 4486.

Before the images can be further analyzed, the sky background must be subtracted. Unfortunately, this cannot be determined directly from the images, since light from the target galaxy fills all four PC chips. Nevertheless, we were able to measure the sky background from an image of a blank field at the same heliocentric ecliptic coordinates as the Virgo cluster. (Zodiacal light, the main source of sky background is a function of heliocentric ecliptic coordinates.) The adopted background is $(3.8 \pm 0.9) \times 10^{-3}$ counts per second. This value was appropriately scaled by the exposure time and subtracted from the images of all the galaxies. Uncertainties in the determination of the sky background should not be a significant source of error for our data, since in all cases the 
sky background is negligible compared to the central regions of the galaxies.

\subsection{Image Deconvolution and Analysis}

All PC images are blurred by the spherical aberration in the HST primary mirror. Nonetheless, the aberrated point spread function (PSF) has a sharp core containing spatial information up to a spatial frequency of 10 cycles $\operatorname{arcsec}{ }^{-1}$, and is stable. As will be shown in Papers II and III, these characteristics of the PSF allow us to partially reconstruct our high signal to noise images by means of deconvolution algorithms with high reliability.

For our deconvolutions we used theoretical PSFs created by means of the TINYTIM software developed by the Space Telescope Science Institute (Krist 1992). The model PSF is noise free and depends on the instrument and filter used, the $H S T$ main optics focal position on the date of observation, the spectral shape of the source, and the position of the image on the CCD. Burrows et al. (1991) showed that the agreement of these model PSFs with the observed PSFs is very good. Our experiments with deconvolution also show the model PSFs to give better results than the (noisy) observed PSFs. We have employed two different reconstruction methods: the Richardson-Lucy (RL) algorithm (Lucy 1974; Snyder 1990), as implemented in the STSDAS package in IRAF, and a Fourier filtering algorithm (see Paper II). Both methods suffered from boundary problems. Therefore, the outer 50 lines and columns are removed, after deconvolution, from the full $800 \times 800$ image.

In order to test the reliability of our deconvolution techniques, we have performed a large number of tests on simulated galaxies. These are described in detail in both Paper II and Paper III. Briefly, the conclusions from the simulations are these:

(1) The RL and Fourier reconstruction methods yield equivalent results. We base most of the rest of this paper on the RL algorithm, using 100 iterations.

(2) The simulations demonstrate that reliable information on the surface brightness profile is recoverable on scales $r \geqq 0$ ". 2 , while reliable information on the other parameters describing the isophotal shapes is recoverable for $r \geqslant 0$ ". 5 .

(3) The results are not sensitive to PSF changes on the scales expected from spacecraft pointing jitter or variations with position in the focal plane.

(4) At radii $\gtrsim 2$ ", where the atmospheric seeing permits them, comparisons with ground-based studies are good.

It is worth pointing out that even in the presence of spherical aberration, the HST is capable of galaxy surface photometry on scales about a factor of ten smaller than has been achieved with ground-based telescopes.

\section{THE IMAGES}

In this section we present the images and discuss those features that are evident without more sophisticated analysis. This deeper analysis is presented in detail in Papers II and III, and conclusions are summarized in Sec. 5 below.

Two greyscale images for each galaxy are shown in Figs. 1 and 2 . For each galaxy the first image is the RL deconvo- lution of the $H S T$ data, rotated so that North is up and East is left. A region of $11.2 \times 11$ ". 2 , or $800 \times 800 \mathrm{pc}$, is shown. The corresponding image in Fig. 2 shows scans from the Space Telescope Science Institute Guide Star Selection System (GSSS) "Quick J" plates taken with the Palomar 48 " Schmidt telescope. These images are included here to illustrate the galaxy morphology at larger radii and to indicate the cluster environment surrounding each galaxy. The GSSS images cover $21^{\prime} \times 21^{\prime}$, are oriented parallel to the HST images, and are centered on the program galaxies. The image of NGC 4478 was omitted because it is visible on the images of NGC 4486 and NGC 4476.

In 5 of the 14 galaxies dust is clearly visible in the deconvolved images. In the strong radio galaxy NGC 4261 we found a sharply defined, elliptical dust disk with a major axis of 1.7 (120 pc) oriented perpendicular to the radio axis [Kormendy \& Stauffer 1987; see Jaffe et al. $(1993,1994)$ for an extensive discussion of this galaxy]. In NGC 4476 a large dust ring $\left(r \approx 12^{\prime \prime}\right)$ is visible with a rather clumpy structure. In the radio galaxy NGC 4374 a complicated dust lane is visible projected against the nucleus. The absorption "striations" have an appearance which is similar to the warped, nearly edge-on captured disk in NGC 5128 (Centaurus A). The dust in NGC 4374 may represent a small, warped disk which will eventually settle into a principal plane of the triaxial potential and reach an equilibrium similar to the disk in NGC 4261. As in Cen A and NGC 4261, the dust lane is perpendicular to the radio axis. Morphologically similar disks are also seen in nonradio galaxies (Zeilinger 1994). In two other galaxies, NGC 4342 and NGC 4550, the dust is filamentary and has not been detected before.

In NGC 4472 a very faint radial dust feature is visible at radii 0.3 to $1^{\prime \prime} 5$ at position angle $140^{\circ}$. Unfortunately, this feature is less clear in the reproductions in Fig. 1 than in the original processed image. Similar very faint patches of dust can be seen on the original images in NGC 4564, NGC 4186, and NGC 4623. In the image of NGC 4486 we do not detect the low contrast dust features seen from the ground (Sparks et al. 1993; Zeilinger et al. 1993) because of the low signal/ noise ratio of our image.

NGC 4486, NGC 4261, NGC 4476, and possibly NGC 4374 show bright unresolved nuclei. The last case is uncertain because of dust obscuration. A number of the fainter galaxies, such as NGC 4570, NGC 4342, and NGC 4623 show well resolved nuclear structures with components that are much flatter than the surrounding elliptical body. NGC 4486 shows its famous jet.

Thus, superficial examination of images with a spatial resolution of $\sim 10 \mathrm{pc}$ indicates that most early galaxies have detailed structure, be it dust, point nuclei, or disky objects, in their centers. However, none of the three systems known to contain kinematically distinct components (NGC 4365, NGC 4472, and NGC 4550) show morphological anomalies that might be linked to these components.

\section{RESULTS}

In Papers II and III we examine the morphology of the centers of these objects quantitatively and relate it to their 


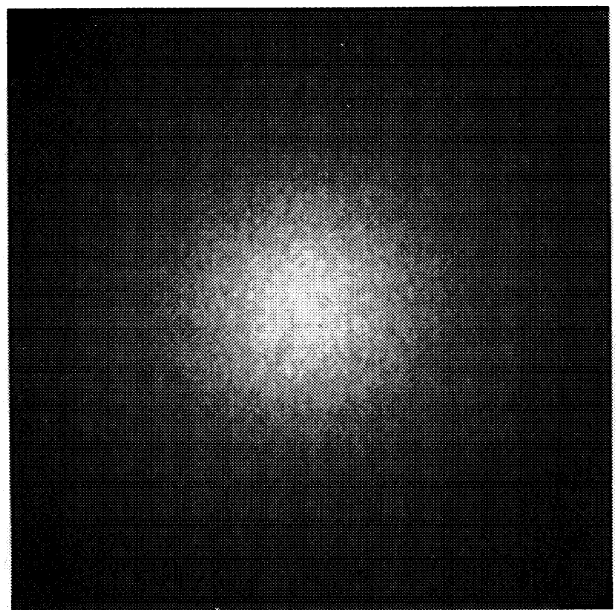

(a)

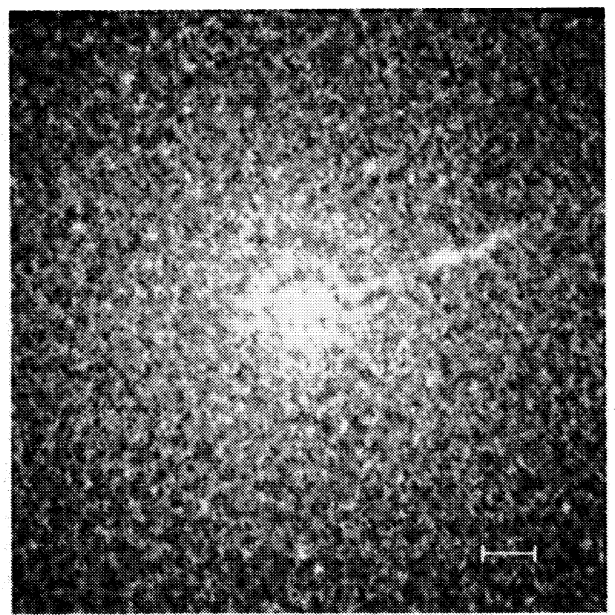

(b)

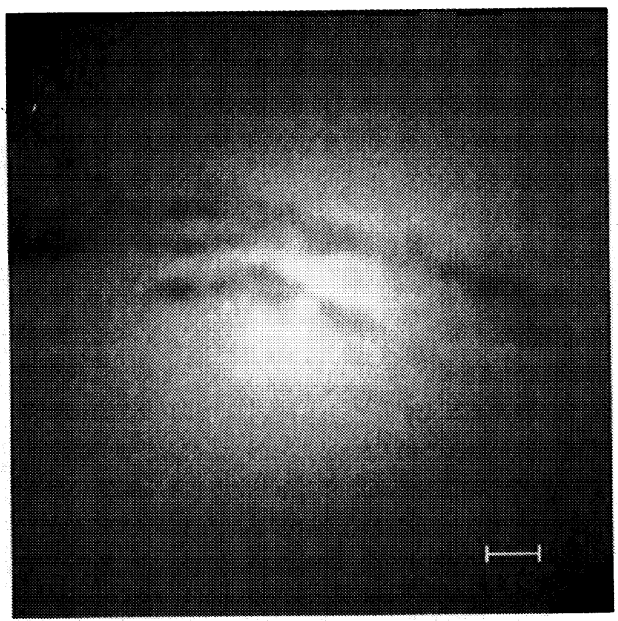

(c)

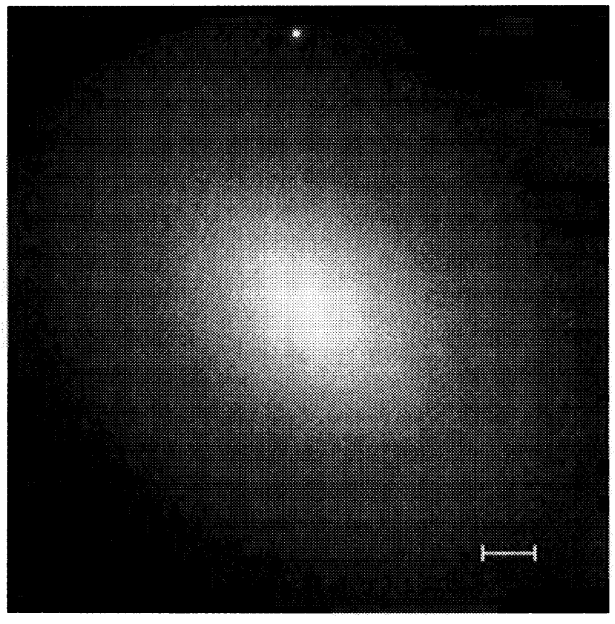

(d)

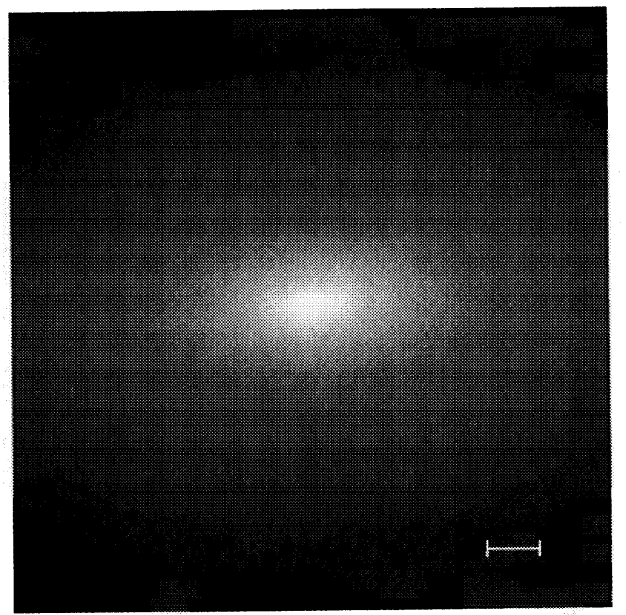

(e)

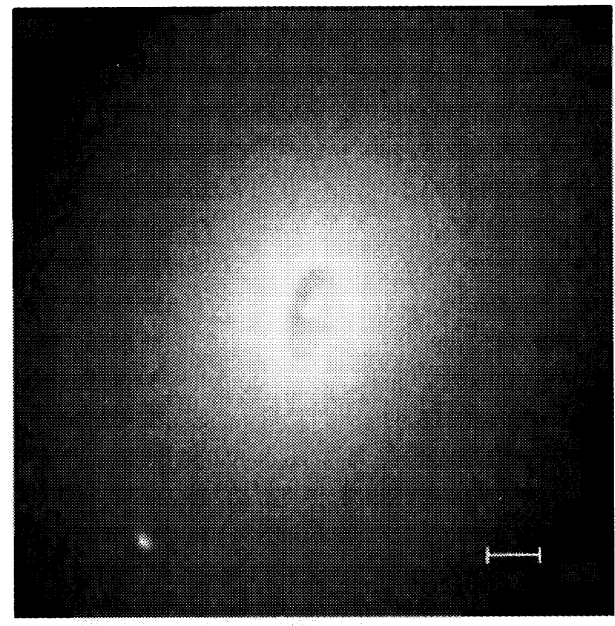

(f)

FIG. 1. HST PC image of the nucleus of the galaxy. The bar in the lower right corner represents $1^{\prime \prime}$; the field of view is 11 .'2. (a) NGC 4472 . (b) NGC 4486 . (c) NGC 4374. (d) NGC 4365. (e) NGC 4473. (f) NGC 4261. 


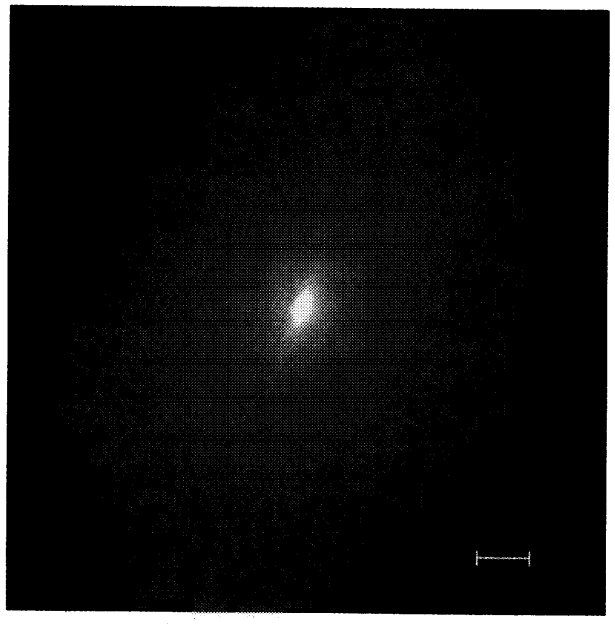

(g)



(h)

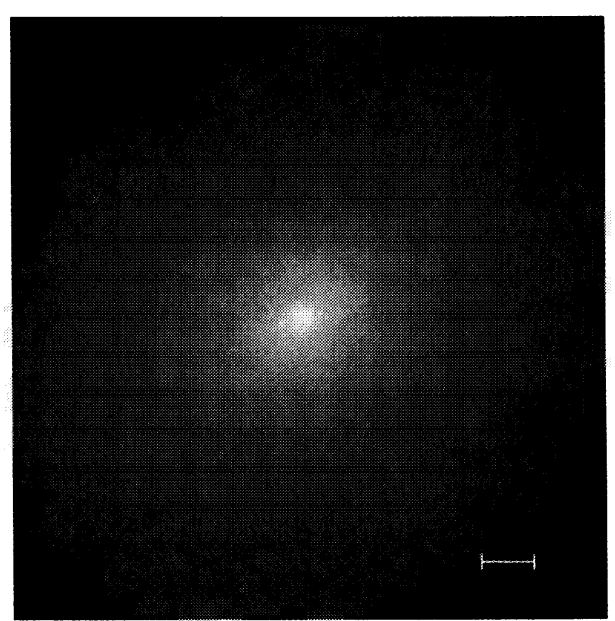

(i)

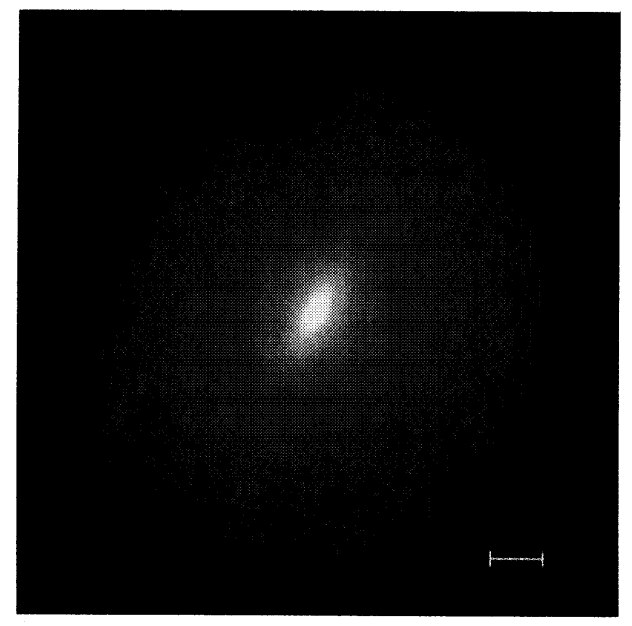

(j)

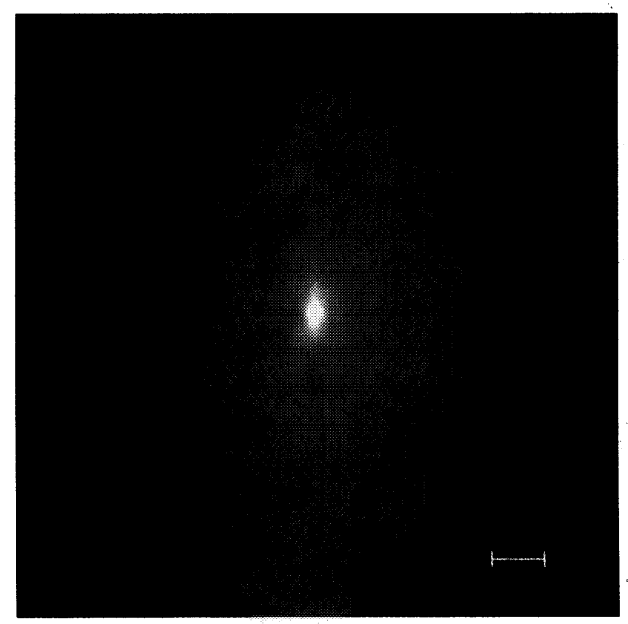

(k)

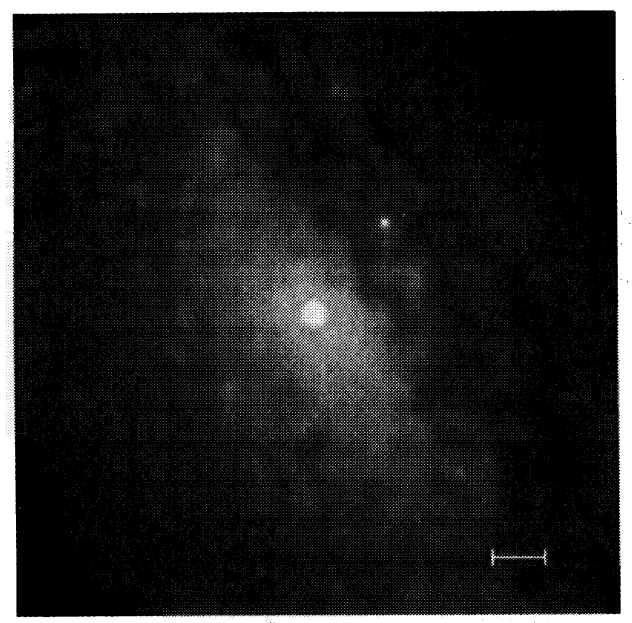

(l)

Fig. 1 (continued). (g) NGC 4570. (h) NGC 4564. (i) NGC 4168. (j) NGC 4478. (k) NGC 4550. (l) NGC 4476. 


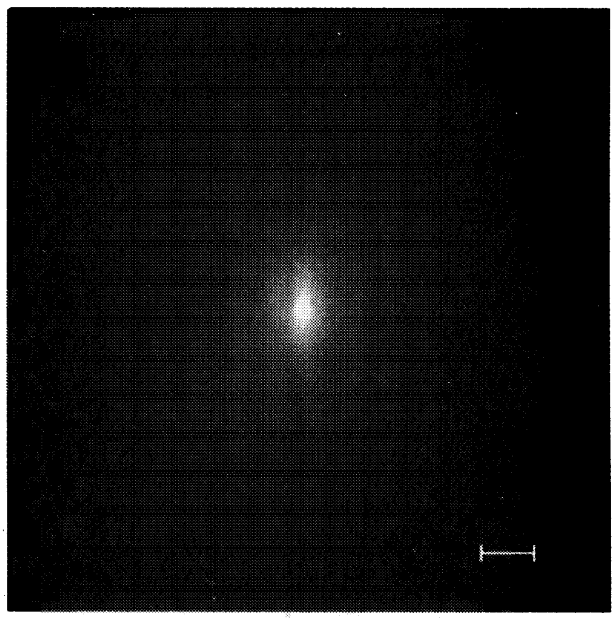

(m)

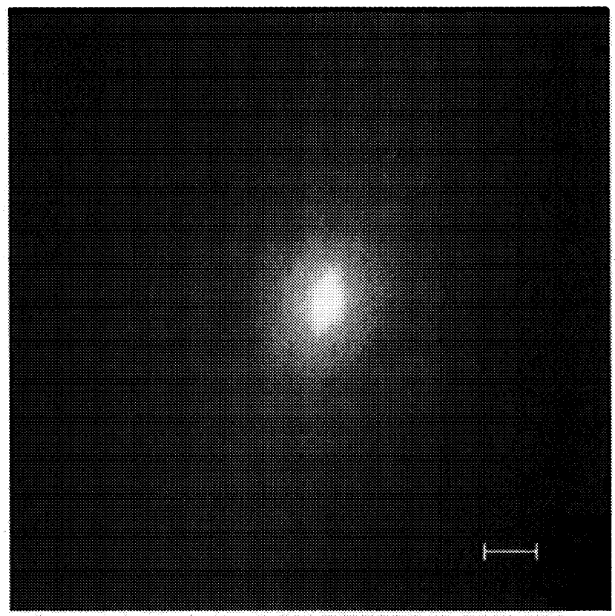

(n)

Fig. 1 (continued). (m) NGC 4623. (n) NGC 4342.

global properties. The most important characteristics found there, including a dimorphic type classification, are listed in Table 2 for each galaxy in the sample.

\subsection{The Dimorphism of Early Type Galaxies}

On the basis of their shape and brightness profiles, our sample of objects (all classified as "E" or "E/SO" on the RSA system) divides into two subtypes, which we call Type I and Type II. Type I galaxies, the classical luminous ellipticals, are distinguished by the following characteristics:

(1) Their luminosity profiles can be fitted accurately with a double power law for radii smaller than $\sim 15^{\prime \prime}$, corresponding to approximately $1 \mathrm{kpc}$ at the distance of Virgo. Although the luminosity profiles flatten at small radii towards a shallow power law, none of these cores are isothermal. The inner power law profile continues to the smallest measurable radius $(0$ " $2=14 \mathrm{pc})$, but the slope of this central "cusp" is quite small, $\leqslant 0.4$.

(2) In general, Type I galaxies are luminous systems $\left(B_{T}<11\right.$ which corresponds to $\left.M_{B}<-20\right)$ with a relatively low central surface brightness: typically $17>\mu_{V}>16$ mag $\operatorname{arcsec}^{-2}$ in the F555W band.
(3) Their ellipticity is low $(<0.4)$ and fairly constant with radius, so that the profiles along the major and minor axes are quite similar. The RSA classifications of the Type I systems in our sample are E0-3. Their isophotes are usually found to be slightly boxy.

(4) All the active galaxies in our sample (NGC 4261, NGC 4374, NGC 4486, and NGC 4472) belong to Type I.

Type II galaxies are generally less luminous than type I galaxies and otherwise have a complementary set of properties. They appear to be composite systems, containing a bulge, an "outer disk" on scale sizes of $100-1000 \mathrm{pc}$, and often a distinct bright nuclear disk with a radius of about 100 pc. Because of the disk components their isophotes are flattened, disky, and show large ellipticity gradients. Some, though not all, of the Type IIs in our sample are classified as S0's in the RC2 system. Type II brightness profiles show complex structure and continue rising steeply to the smallest radii measurable with the $H S T$.

This dichotomy of early type galaxy morphology corresponds at least roughly to distinctions made earlier by other authors from "macroscopic" (kpc scale) criteria observed from the ground. This is discussed in some detail in Paper II, Sec. 3.4. Capaccioli, et al. (1993) convincingly demonstrate dichotomy in a survey of 1500 galaxies. They refer to the two classes as "bright" and "ordinary," and show that the two classes have distinct distributions of almost all macroscopic observable parameters, including diskiness, ellipticity, metallicity, degree of rotational support, presence of cool gas, and nuclear activity. They find the two types to separate at $M_{B} \sim-19.3$. Adjusted to our assumed distance to Virgo, this corresponds to $B_{T} \sim 12$.

It is clear that our Type II galaxies correspond in large measure to the "ordinary" systems having rapid rotation and some evidence of lenticular morphology. Since our definition is based primarily on morphological criteria visible at radii of $100-1000 \mathrm{pc}$, while the other classifications are based on scale sizes ten times larger and on kinematic information, the question of whether these classes are in fact identical can only be decided by a future comparison of their properties on these different scales.

It is interesting that in both Type I and Type II galaxies a radius of $\sim 100-200 \mathrm{pc}$ seems to be a specific characteristic size: that of the nuclear disks for Type II galaxies and of the core radius of Type I galaxies.

Within the limited size of our sample we find no correlation of core size or central surface brightness with galaxy luminosity for Type I galaxies (cf. Paper III). The correlation found in earlier ground based surveys (Lauer 1985; Kormendy \& Djorgovski 1989) may be the result of including morphologically distinct types such as cluster cDs and dwarf ellipticals at the extreme luminosities. In this regard, the somewhat larger core radius of NGC 4486 (M87) could be explained by its position as a dominant cluster galaxy.

All of the galaxies with strong radio $(20 \mathrm{~cm})$ or $\mathrm{x}$-ray (between 0.5 and $4.5 \mathrm{keV}$ ) emission are Type I. These measures of activity correlate with galaxy mass, so this result is not surprising. It is in agreement with the findings of Bender et al. (1989), and Capaccioli et al. (1993), who show that 


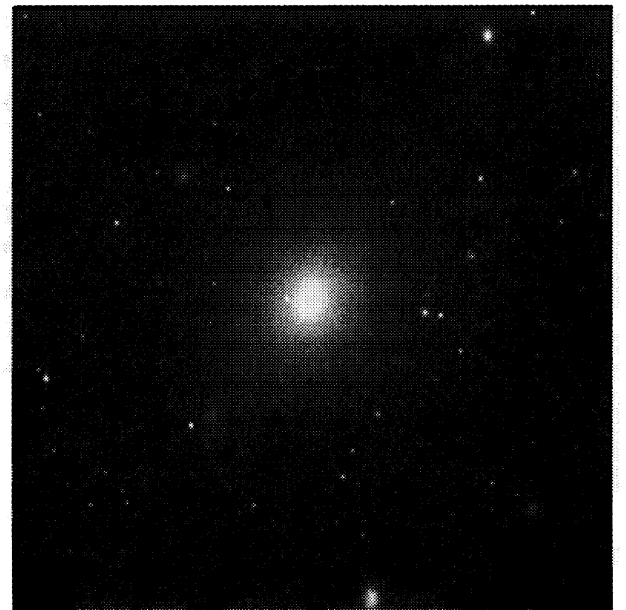

(a)

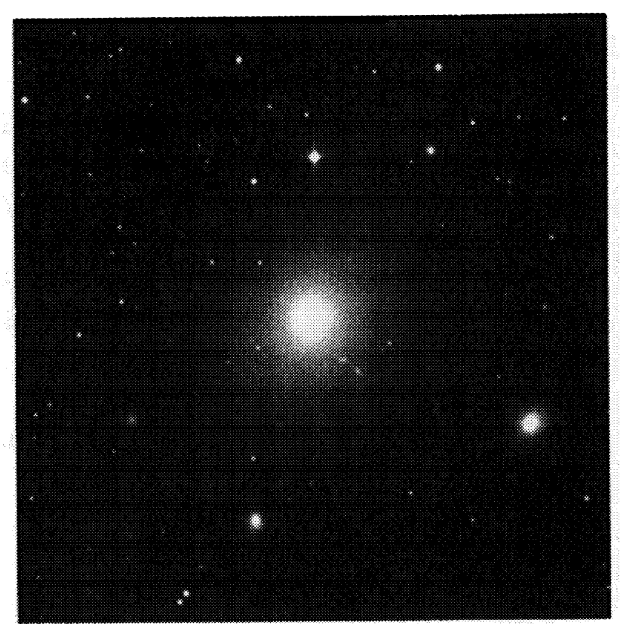

(b)

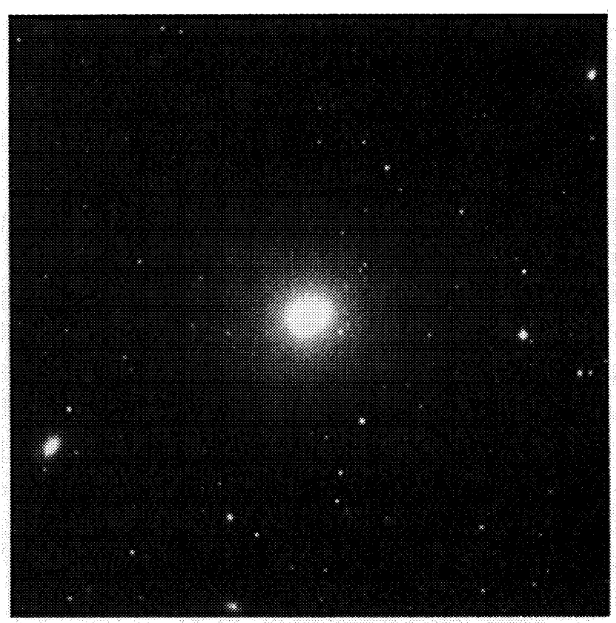

(c)

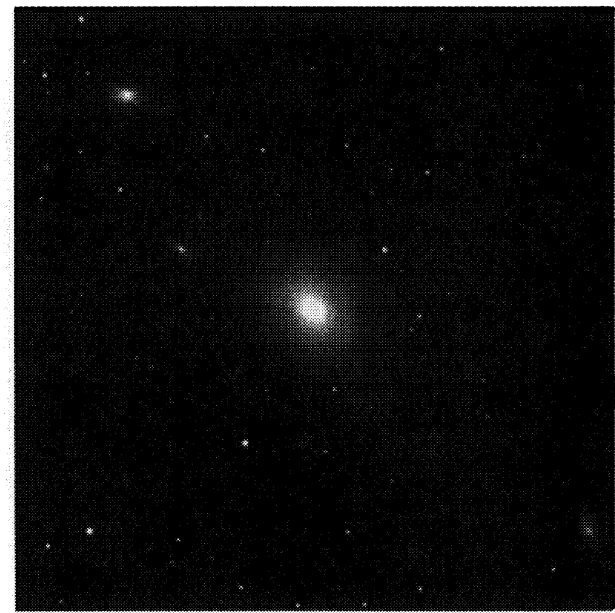

(d)

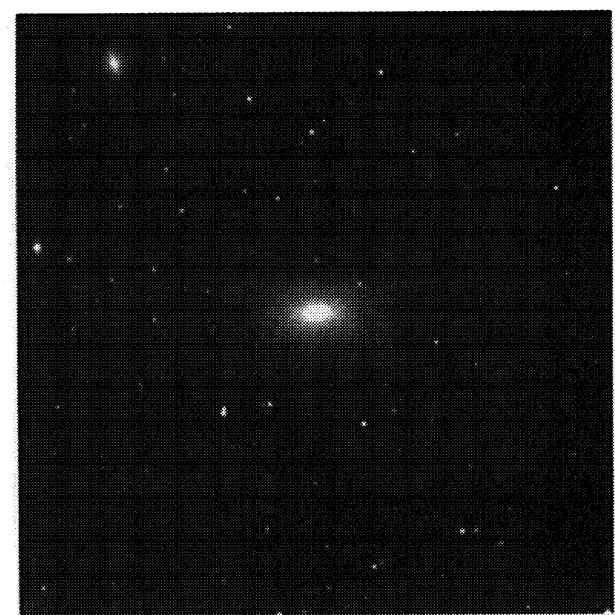

(e)

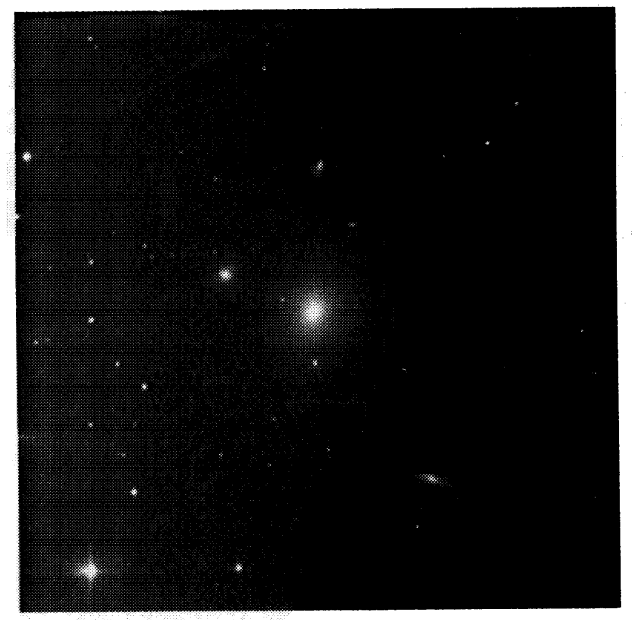

(f)

FIG. 2. Digitized scan of the STScI GSSS “Quick-J" Sky Survey image surrounding the galaxy. The field of view is 21 '. (a) NGC 4472 . (b) NGC 4486 . (c) NGC 4374. (d) NGC 4365. (e) NGC 4473. (f) NGC 4261. 


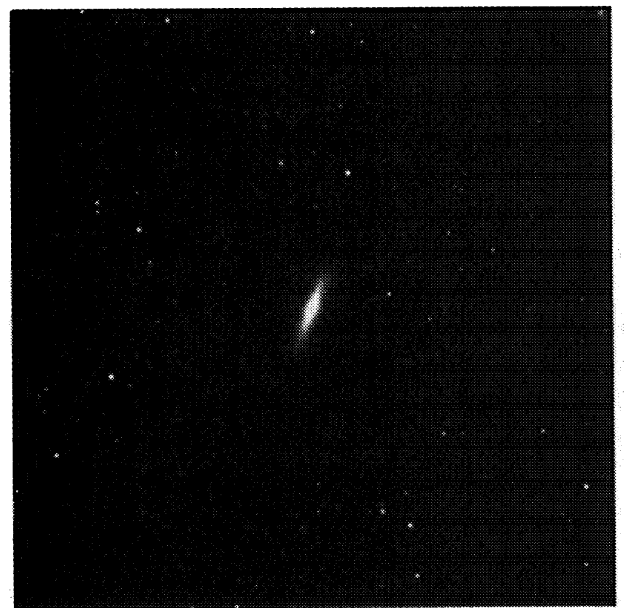

(g)

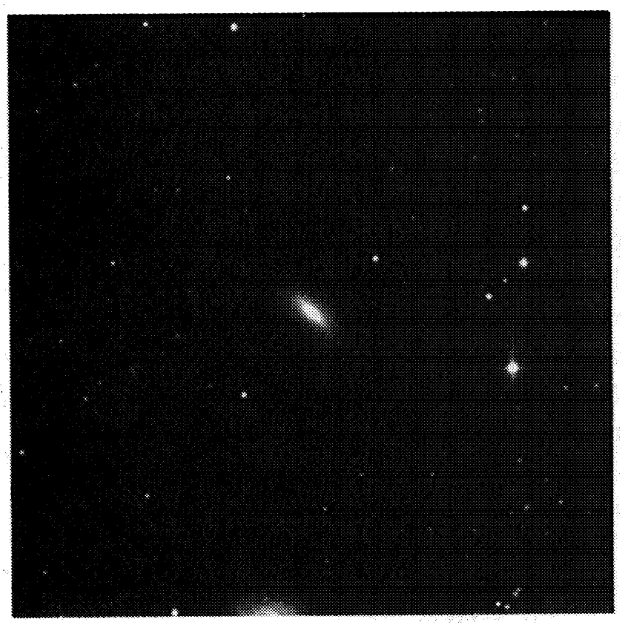

(h)

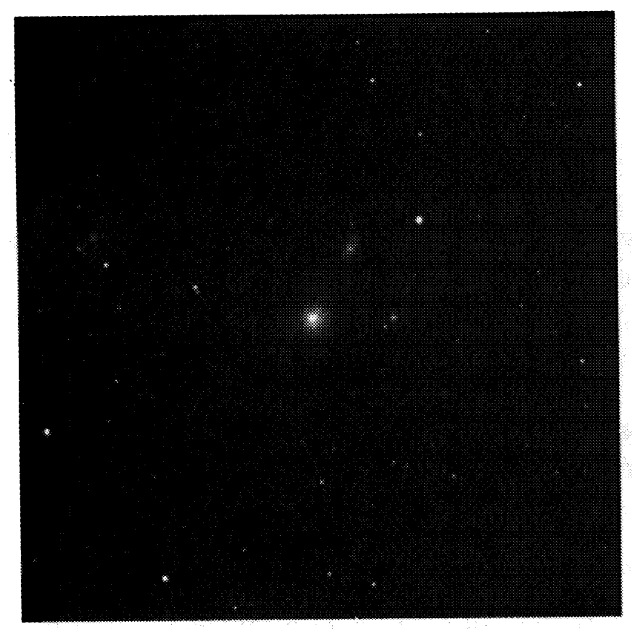

(i)

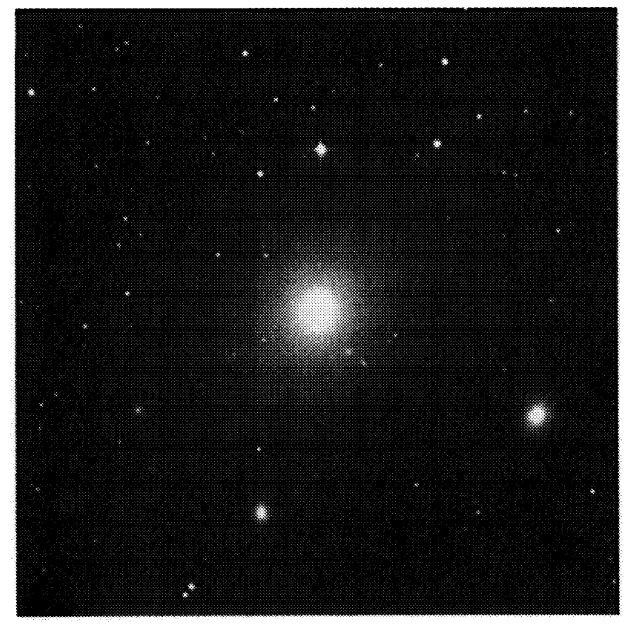

(j)



(k)

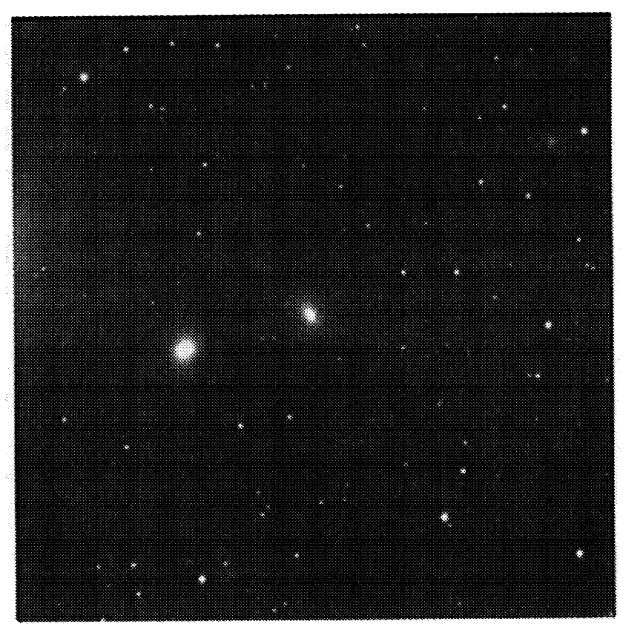

(I)

Fig. 2 (continued). (g) NGC 4570. (h) NGC 4564. (i) NGC 4168. (j) NGC 4478 omitted; galaxy visible on (b). (k) NGC4550. (l) NGC 4476.

(C) American Astronomical Society - Provided by the NASA Astrophysics Data System 


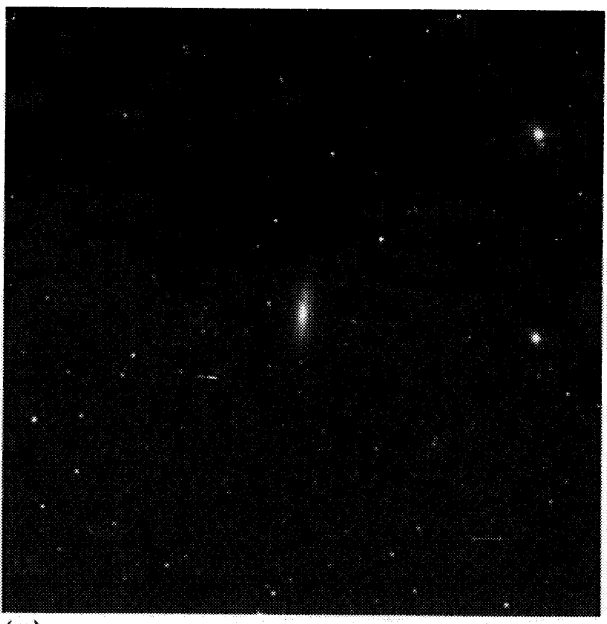

(m)

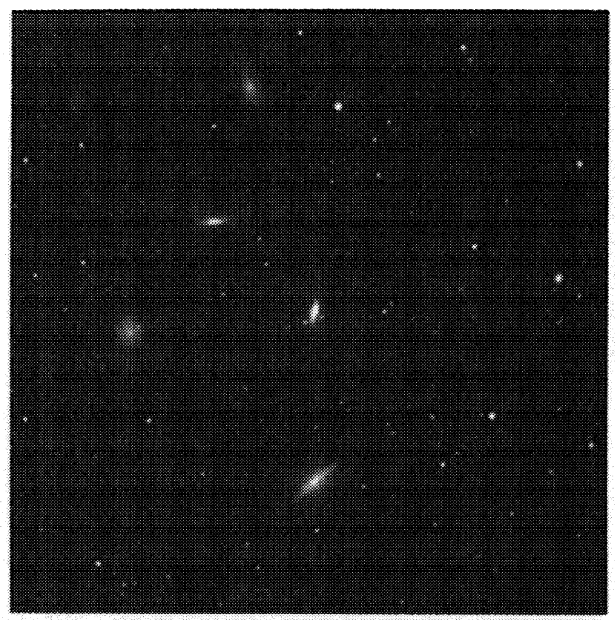

(n)

Fig. 2 (continued). (m) NGC 4623. (n) NGC 4342.

radio and $\mathrm{x}$-ray emission are generally not found in galaxies with disky isophotes.

The dynamically cold stellar disks and highly flattened structures of Type II galaxies imply that they were formed under conditions when gas phase dissipation was important and where the specific angular momentum was fairly high. The persistence of the cold disks, particularly the extremely thin nuclear disks whose scale heights are less than $25 \mathrm{pc}$, indicate that these galaxies have not undergone major dynamical disturbances since their creation (Toth \& Ostriker 1992).

The Type I ellipticals are rounder, more relaxed, and are a magnitude or two brighter, suggesting that either they are formed by the merger of several Type II galaxies (e.g., Barnes \& Hernquist 1992) or that they were formed under very different conditions, where the average specific angular momentum was low and gas dissipation was less important. The coincidence of the characteristic nuclear sizes of the two classes perhaps favors the first explanation.

\subsection{Where are the Face-On Type II Galaxies?}

Most of the characteristics listed above that discriminate Type I from Type II are explained by disk components seen at high inclination angles. For example, the higher central surface brightness in Type II systems is caused by the nuclear disk seen close to edge-on, and the difference between major and minor axes by the foreshortening of the disk components along the minor axis, so that the bulge dominates at larger radii. If one of these galaxies were viewed face-on, it would appear much more like a Type I galaxy; the disk components would drop in brightness (by $1.2 \mathrm{mag}$ for a disk seen initially $20^{\circ}$ from edge-on), and the asymmetries between major and minor axes would disappear. Of all the signatures discussed in Papers II and III that distinguish these classes, only the total luminosity is not affected by inclination. If the bulge component of a Type II galaxy has a luminosity profile that can be fitted by a double power law, it would be classified as a fainter Type I galaxy when viewed face-on.

The distribution of the ellipticities of the Type II galaxies leads us to believe that this has in fact happened. Where an isotropically distributed ensemble of very flat systems would have a uniform distribution in ellipticity, our clear Type II galaxies (NGC 4342, NGC 4570, NGC 4564, NGC 4550, and NGC 4623) all have ellipticities between 0.4 and 0.7 . Again assuming isotropy, an approximately equal number of such systems should have ellipticities below 0.4 , and it is natural to identify the fainter galaxies that we have classified as Type I or intermediate (e.g., NGC 4168, 4473) as in fact Type II systems seen nearly face-on. We therefore provisionally conclude on the basis of our admittedly small sample that essentially all fainter $\left(B_{T}>11\right.$ at Virgo) early type galaxies are of Type II. Only NGC 4168 appears doubtful in this context. While its surface photometry is consistent with either a Type I or face-on Type II, the galaxy shows a low level of $x$-ray, FIR, and radio activity not seen in any unambiguous Type II galaxies.

Van den Bergh (1990) studied the ellipticity distribution of all the S0 galaxies in the RSA catalog and arrived at a similar conclusion: all fainter early type galaxies are in fact lenticulars.

\subsection{Dust in the Nuclei of Early Type Galaxies}

In Sec. 4 we found dust in nine galaxies. In Sec. 3.6 of Paper II we find indications for dust patches in an additional two galaxies. Thus, we find dust in the nucleus of almost every galaxy: $11 / 13$ or $12 / 14$ if the ground based data of M87 is included. The dust occurs with equal frequency in both Type I and II galaxies, including the Type II galaxies with clear disks. In these last cases it is unlikely that the dust has been captured from a recent collision with a larger late type galaxy. Such collisions would disrupt the cold stellar disks (Ostriker 1990; Toth \& Ostriker 1992). These authors indicate that the mass of individual infalling satellites must be less than $\sim 1 \%$ of the mass of the host, thus roughly $\leqslant 10^{9} \mathscr{b}_{\odot}$, to avoid destruction of the disks. Alternatively, the production of dust by evolved stars within the galaxies themselves may be sufficient to balance the destruction of the dust by spallation.

In some cases, for example in Centaurus $\mathrm{A}$, where the remnant of a late type galaxy is still visible, and in NGC 
TABLE 2. Morphological characteristics determined for the surveyed galaxies.

\begin{tabular}{|c|c|c|c|c|c|c|c|}
\hline $\begin{array}{c}\text { Galaxy } \\
\text { (1) }\end{array}$ & $\begin{array}{c}\text { RSA } \\
(2)\end{array}$ & $\begin{array}{c}\alpha(\mathrm{J} 2000) \\
(3)\end{array}$ & $\begin{array}{c}\delta(\mathrm{J} 2000) \\
(4)\end{array}$ & $\begin{array}{c}\text { Type } \\
(5)\end{array}$ & $\begin{array}{c}\text { Dust } \\
\text { (6) }\end{array}$ & $\begin{array}{c}\text { Disk } \\
(7)\end{array}$ & $\begin{array}{c}\text { Remarks } \\
(8)\end{array}$ \\
\hline NGC 4472 & $\mathrm{E} 1 / \mathrm{S}_{1}$ & 122946.673 & +080000.40 & I & $\mathbf{P}$ & & KDC \\
\hline NGC 4486 & E0 & 123049.282 & +122327.90 & I & F & & $\mathrm{PN}+$ jet \\
\hline NGC 4374 & E1 & 122503.757 & +125312.44 & I & F & & PN? \\
\hline NGC 4365 & E3 & 122428.318 & +071902.23 & I & & & $\mathrm{KDC}$ \\
\hline NGC 4473 & E5 & 122949.010 & +132545.74 & II: & P? & & \\
\hline NGC 4261 & E3 & 121923.145 & +054927.02 & I & D & & PN \\
\hline NGC 4570 & $\mathrm{~S}_{1} / \mathrm{E} 7$ & 123653.340 & +071446.18 & II & P? & + & \\
\hline NGC 4564 & E6 & 123627.053 & +112619.01 & II & $\mathbf{P}$ & & \\
\hline NGC 4168 & $\mathrm{E} 2$ & 121217.240 & +131217.30 & I & $\mathbf{P}$ & & \\
\hline NGC 4478 & $\mathrm{E} 2$ & 123017.467 & +121941.16 & II: & & & \\
\hline NGC 4550 & $\mathrm{E} 7 / \mathrm{SO}_{1}$ & 123530.623 & +121313.40 & II & F & & Kin. Disks \\
\hline NGC 4476 & E5pec & 122959.172 & +122053.42 & II: & A & & PN \\
\hline NGC 4623 & E7 & 124210.558 & +074036.98 & II & $\mathbf{P}$ & + & \\
\hline NGC 4342 & E7 & 122339.003 & +070313.52 & II & $P$ & + & \\
\hline
\end{tabular}

Notes to TABLE 2

Column (1) gives the NGC name. Column (2) gives the RSA classification. Columns (3) and (4) give the J2000 position of the isophotal center of the galaxy in the coordinate system defined by the HST guide stars. Typical absolute accuracy is about 0.5. Column (5) gives the Type classification as I (elliptical) or II (disky) as determined in Paper III. A colon indicates an uncertain classification. Column (6) indicates whether dust has been found. The symbols are: D: disk; F: filaments; A: annulus; P: small patch; P?: probable small patch. Column (7) indicates the presence of a nuclear stellar disk (Paper II) and Column (8) includes other remarks: $\mathrm{KDC}=$ Kinematically Decoupled Core; PN=Point Nucleus. NGC 4550 has counterrotating stellar disks on $10^{\prime \prime}$ scale.

4374, which resembles it, capture is the preferred explanation. In others, such as NGC 4365 , where the dust is very smoothly distributed, retention of locally produced dust is more likely. The galaxies showing small patches probably acquire dust by both processes.

In our sample, we see no general relation between nuclear activity and the amount or morphology of dust. While the massive Type I galaxies NGC 4261 and NGC 4374 with large dust systems are indeed active, the modestly active NGC 4472 and the hyperactive NGC 4486 only show small patches. In the two dusty radio galaxies, the dust lane is perpendicular to the radio jets even in NGC 4261, where the radio axis is perpendicular to the galactic spin axis (Jaffe et al. 1993). We also find dust in most of the nonactive Type II galaxies.

Excess. far infrared emission (FIR) is often an indication of dust in galaxies. IRAS $100 \mu \mathrm{m}$ fluxes for the survey galaxies (Knapp et al. 1989), are tabulated in Table 1. The obviously dusty galaxies of both types, NGC 4486, NGC 4374, NGC 4261, NGC 4550, and NGC 4476 all show significant FIR emission. On the other hand, two galaxies showing little or no visible dust features and no nuclear radio emission, NGC 4365 and NGC 4168, have similar FIR fluxes. A similar situation in the bulge of M31 (Soifer et al: 1986) was explained by a very smooth, weak, diffuse, dust component generated in the atmosphere of evolved bulge stars. This dust reemits absorbed star light in the FIR without revealing itself in patchiness or color gradients. This explanation is plausible for these two galaxies in our survey: the FIR fluxes and optical luminosities are comparable to the bulge of M31, and the FIR fluxes are consistent with the absorption and reemission of a few percent of the visible light. It is puzzling that the more massive galaxy NGC 4472 shows no equivalent
FIR flux, but the dense $x$-ray halo of this galaxy may rapidly destroy dust by sputtering.

We note that ground based indicators of cool interstellar matter, such as FIR emission only pick up the very largest of the dust systems we detect visually.

\section{CONCLUSIONS}

(1) The dimorphism of early type galaxies discerned from ground based criteria is strikingly confirmed by observations of their nuclei. "E" galaxies are divided into Type I ("true" ellipticals) and Type II ("disky" ellipticals). None of the Type I galaxies have isothermal cores, and the brightness profiles of the Type II galaxies continue rising approximately as power laws to the $H S T$ resolution limit. The nuclei of Type II galaxies are often distinguished by thin stellar disks with radii of $\sim 100 \mathrm{pc}$. In these cases, the outer disks seem to show an inner edge at a larger radius, leaving a distinct gap between the two disks.

(2) Essentially all early type galaxies fainter than $M_{B} \simeq-20$ are Type II ("disky" or lenticular); there appear to be no Type I (true elliptical) galaxies flatter than E4.

(3) The Type I galaxies typically show power law brightness profiles in the cores, i.e., cusps, but the logarithmic slopes of these profiles are quite flat, typically $\sim 0.2$.

(4) Almost all galaxies in our sample have dust in their nuclear region, irrespective of classification or level of nuclear activity. However, the central dust disk in NGC 4261 , is a striking association between the interstellar medium of an elliptical galaxy and nuclear activity.

(5) The three systems in our sample with kinematic evidence of distinct components exhibit no photometric anoma- 
lies that can be associated with these subsystems.

(6) For bright, noncluster dominant ellipticals the correlation between luminosity and core radius appears weak.

H.C.F., L.F., and R.W.O. received partial support from NASA/STScI Grant No. GO-2607.01-87A, and R.W.O.'s work was also supported in part by NASA Long Term Space Astrophysics Grant No. NAGW-2596. F.C.vdB. acknowledges support from the Netherlands Foundation for Astronomical Research (ASTRON) (Grant No. 782-373-055) with financial aid from the Netherlands Organization for Scientific Research (NWO).

\section{REFERENCES}

Barnes, J. E., \& Hernquist, L. E. 1992, ARA\&A, 30, 705

Bender, R., Surma, P., Döbereiner, S., Möllenhoff, C., \& Madejsky, R. 1989, A\&A, 217, 35

Burrows, C. J., Holtzman, J. A., Faber, S. M., Bely, P. Y., Hasan, M., Lynds, C. R., \& Schroeder, D. 1991, ApJ, 369, L21

Capaccioli, M., Caon, N., \& D’Onofrio, M. 1993, in Structure, Dynamics, and Chemical Evolution of Elliptical Galaxies, ESO Conference and Workshop Proceedings No. 45, edited by I. J. Danziger, W. W. Zeilinger, and K. Kjär, p. 43

de Vaucouleurs, G., de Vaucouleurs, A., \& Corwin, H. C. 1976, Second Reference Catalogue of Bright Galaxies, (University of Texas, Austin) ( RC2)

Fabricant, D., Lecar, M., \& Gorenstein, P. 1980, ApJ, 214, 552

Ferrarese, L., Van den Bosch, F. C., Ford, H. C., Jaffe, W., \& O'Connell, R. W. 1994, AJ, 108, 1598 (Paper III)

Huchra, J. P. 1984, in The Virgo Cluster of Galaxies, ESO Conference and Workshop Proceedings No. 2, edited by O.-G. Richter and B. Binggeli, p. 181

Jacoby, G. H., Ciardullo, R., \& Ford, H. C. 1990, ApJ, 356, 332

Jaffe, W., Ford, H. C., Ferrarese, L. van den Bosch, F. C., \& O'Connell, R. W. 1993, Nature, 364, 213

Jaffe, W., Ford, H. C., Ferrarese, L. van den Bosch, F. C., \& O'Connell, R. W. 1994 (in preparation)

Knapp, G. R., Guhathakurta, P., Kim., D.-W., \& Jura, M. 1989, ApJS, 70, 329

Kormendy, J. 1984, ApJ, 287, 577

Kormendy, J. 1993, in The Nearest Active Galaxies, edited by J. E. Beckman, H. Netzer, and L. Colina (Consejo Superior de Investigaciones Scientificas, Madrid) (in press)

Kormendy, J., \& Djorgovski, S. 1989, ARA\&A, 27, 235

Kormendy, J., \& Stauffer, J. 1987, IAU Symposium No. 127, Structure and Dynamics of Elliptical Galaxies, edited by T. De Zeeuw (Reidel, Dordrecht)

Krist, J. 1992, The Tiny Tim User's Manual, Version 1.0, STScI publications Lauer, T. R. 1985, ApJ, 292, 104
Lauer, T. R. 1989, PASP, 101, 445

Lauer, T. R., et al. 1992a, AJ, 104, 552

Lauer, T. R., et al. 1992b, AJ, 103, 703

Lauer, T. R., et al. 1993, AJ, 106, 1436

Lucy, L. B. 1974, AJ, 74, 745

MacKenty, J. W., et al. 1992, Wide Field and Planetary Camera Instrument Handbook, Version 3.0 (STScI, Baltimore)

O'Connell, R. W. 1994, in Nuclei of Normal Galaxies: Lessons from the Galactic Center, edited by R. Genzel (Kluwer, Dordrecht) (in press)

Ostriker, J. P. 1990, in Evolution of the Universe of Galaxies, Astronomical Society of the Pacific Conferences Series, Volume 10, edited by R. G. Kron (San Francisco), p. 25

Rees, M. J. 1984, ARA\&A, 22, 471

Roberts, M. S., Hogg, D. E., Bregman, J. N., Forman, W. R., \& Jones, C. 1991, ApJS, 75, 751

Sandage, A., \& Tammann, G. A. 1981, A Revised Shapley-Ames Catalogue of Bright Galaxies (Carnegie Institution of Washington, Washington)

Snyder, D. L. 1990, in The Restoration of HST Images and Spectra, edited by R. L. White and R. J. Allen (STScI, Baltimore), 56

Soifer, B. T., Rice, W. L., Mould, J. R., Gillett, F. C., Rowan-Robinson, M. \& Habing, H. J. 1986, ApJ, 304, 651

Sparks, W. B., Ford, H. C., \& Kinney, A. 1993, ApJ, 413, 531

Stiavelli, M., Møller P., \& Zeilinger, W. W. 1993, A\&A, 277, 421

Toth, G., \& Ostriker, J. P. 1992, ApJ, 389, 5

Van den Bergh, S. 1990, ApJ, 348, 57

Van den Bosch, F. C., Ferrarese, L., Jaffe, W., Ford, H. C., \& O'Connell, R. W. 1994, AJ, 108, 1579 (Paper II)

White, R. L., \& Allen, R. J. 1990, The Restoration of HST Images and Spectra (STScI, Baltimore)

Wrobel, J. M. 1991, AJ, 101, 127

Young, P. J. 1980, ApJ, 242, 1232

Zeilinger, W. W. 1994, ST-ECF Newsletter, 21, 29

Zeilinger, W. W., Møller, P., \& Stiavelli, M. 1993, MNRAS, 261, 175 